\title{
Humanoid Nurse Robots as Caring Entities: A Revolutionary Probability?
}

\author{
Rozzano C. Locsin ${ }^{1}$, Hirokazu Ito ${ }^{2}$, Tetsuya Tanioka ${ }^{2}$, Yuko Yasuhara ${ }^{2}$, Kyoko Osaka ${ }^{2} \&$ Savina O. Schoenhofer ${ }^{3}$ \\ ${ }^{1}$ Institute of Biomedical Sciences Graduate School, Tokushima University, Tokushima, Japan; Florida Atlantic \\ University, Boca Raton, USA \\ ${ }^{2}$ Institute of Biomedical Sciences Graduate School, Tokushima University, Tokushima, Japan \\ ${ }^{3}$ Anne Boykin Institute, Florida Atlantic University, Boca Raton, USA \\ Correspondence: Rozzano C. Locsin, Professor of Nursing, Institute of Biomedical Sciences Graduate School, \\ Tokushima University, Tokushima, Japan and Professor Emeritus, Florida Atlantic University, Boca Raton, USA.
}

Received: July 27, 2018

doi:10.20849/ijsn.v3i2.456
Accepted: August 9, $2018 \quad$ Online Published: August 22, 2018

URL: https://doi.org/10.20849/ijsn.v3i2.456

\begin{abstract}
The probability that humanoid nurse robots (HNRs) can be caring seems plausible. The question explored by this article is: what are humanoid nurse robots manifesting when they become more functionally advanced and human-like? Its discussion is grounded in several foundational philosophical and theoretical viewpoints such as Plato's focus on "soul," Rogers' emphasis on human energy fields, Boykin and Schoenhofer's assumption of persons as caring because of their humanness, and the prescriptive or predictive practice of nursing. Imploring logical explanations, the possibility of humanoid robots manifesting caring seems to become more likely. In future developments, to what end will HNRs be functional and able to manifest caring regardless of their human-likeness?
\end{abstract}

Keywords: humanoid nurse robots, practicing nursing, caring, nursing

\section{Introduction}

The revolutionary likelihood of humanoid nurse robots (HNRs) being and becoming able to manifest caring is inspired by philosophical and theoretical viewpoints such as those that Plato's philosophical thoughts inspire. HNRs are robotic technologies with features much like humans and engendered with capabilities. Relying on logical explanations, the questions posited in this article are "What is the nature of HNRs as caring entities?" and "are humanoid nurse robots functionally able to manifest caring?" With this revolutionizing possibility, will the involvement of HNRs in nursing influence human health and well-being?

This discussion paper is grounded in the perspective of nursing as the practice essential to the well-being and health of human persons through knowing persons as caring (Locsin, 2005). Locsin and Purnell (2015) have described three processes of nursing occurring all at once and interacting within the Universal Technological Domain, that is, technological knowing, mutual designing, and participative engaging. These processes may involve HNRs as critical protagonists that are integral to the practice of nursing. In this probabilistic scenario, the design, development and capabilities of HNRs become vital elements, and programming of HNRs with quantum computers (Savage, 2017) and affective computers must be considered indispensable, so that acceptance, confirmation, revelation, and support of persons as caring in nursing may be achieved (Locsin \& Ito, 2018, (Locsin, 2017). In addition, HNRs must possess advanced artificial intelligence, which Barrat (2013) calls artificial super intelligence (ASI), and as declared by Yonck (2017), an affective computer in order to harness the emotive or impassioned aspects of HNRs.

Assuming that HNRs manifest caring may be a far-fetched idea, or if probable, its realization would require the consideration of future accoutrements. Tanioka (2017) has provided a predictive timeline of 2050, in consonance with Kurzweil's (2005) prediction of the technological singularity occurring in 2045, that is, when artificial super intelligence (ASI) will surpass human intelligence, giving legitimacy to the pertinence of human-machine communication, function and robot physiognomy. Being human in relationship with artificially intelligent machines will not only challenge the utilitarian aspects of intelligent machines, but it will also be material to their relationships as machines to human beings. The capacity of HNRs to manifest caring provides credence to 
futuristic predictions of the development and design of intelligent machines that also include theoretical underpinnings, and this capacity will be influential in establishing functional, and perhaps, seamless and seemingly natural human-machine relationships.

This discussion paper acknowledges questions about caring and manifestations of caring, the functionalities of humanoid robots as "beings" which are endowed with a caring capacity by possessing qualities to ably manifest caring. Furthermore, it addresses the (kinds of) illustrations of human-like features that can or may be "installed" in the HNRs that would enable them to manifest caring. Important guidance and direction for intelligent humanoid robots to be significant protagonists in their contribution to the practice of nursing is bestowed by theory-based processes such as knowing persons as caring grounded in the theory of Technological Competency as Caring in Nursing, (Locsin, 2005), (Locsin \& Ito, 2017), the Transactive Relationship Theory of Nursing (Tanioka, 2017), and imbued with viewpoints from Nursing as Caring (Boykin \& Schoenhofer, 2001). With these theories framing HNRs' capabilities to manifest caring, it seems to be more in the realm of probability rather than (un)certainty that their practice can be logically manifested and identified as caring.

In Tanioka's (2017) Transactive Relationship Theory of Nursing, the 'participative engagement' process within the transactive relationship can be manifested in the human-machine interface that signals efficiently functioning HNRs. As an ultimate requirement, it is necessary that nurse scientists, roboticists and computer scientists design and develop HNRs to be programmable functionalities with human-like physiognomies and capable of engaging in affective, emotive, and transactive processes, thereby ensuring a seamless futuristic practice manifesting caring. As suggested by the National Institute of Advanced Industrial Science and Technology of Japan, some basic guidelines of robotic devices for nursing care enhance the revolutionary probability of a humanoid nurse robot as manifesting caring (Robotic devices for nursing care, 2016).

\section{Some Philosophical and Theoretical Fundamental Thoughts Regarding Persons}

"Do HNRs have souls?" quipped a scholar of caring science during one opportune moment in an engaging meeting. The dichotomy and co-existence of the soul and the body seem to assert oneness as material to the understanding of human beings as person. As illumined by Plato and Socrates, in accepting the immortality of the soul as the essence of being human, and often considered to be synonymous with the mind or the self, as declared by Descartes' distinction of the human mind and body (Wilson, 1976) thereby conferring individuality and humanity to humans (Encyclopedia Britannica On-line, 2017, para.1), it is the Linnaean classifications that initially directed the sorting of human beings as homo sapiens, from a biologic-anatomical-physiological perspective. Existing beliefs in the soul moved philosophers to explain its nature, relatedness to the human body, its origin and its impermanence. In this paper, the soul is presented as a reflection of the supposed composition and dichotomous state of life-emphasizing the duality of mind and self, not necessarily that the soul is the essential component of being human.

As such, in order for HNRs to communicate their 'being' as more human-like, communication through natural languages (NLP or natural language processing), like human beings, is necessary. Ren and Matsumoto (2017) and Wagoner and Matson (2015) clearly support and explain these processing capabilities in their studies. Furthermore, databases of HNRs should be enhanced with the kind of multiple learning functions artificial super intelligence is capable of, in order to correspond appropriately and accurately with expressions of embodying the "soul" and its ethico-moral dynamic and human-like frailties.

Nevertheless, the question remains, 'is the soul a critical part of being human?' It is believed that the ability to communicate the essence of being human is more critical than the view of looking more like a human. Any discussion about the soul responds to the question of whether or not HNRs can have a soul. The answer should be an explicit "no, not at all," as the soul is more a theological concept than a naturally or biologically "designed" concept of being human. In addition, from a theological and philosophical view, St. Augustine declared that the soul is a 'rider' of the body, clarifying the separation of the material and irrelevance of the soul and the being of the person (Encyclopedia Britannica On-line, 2017, para. 6), while the Muslim concept, like that of Christianity, "holds that the soul comes into existence at the same time as the body. Thereafter, it has a life of its own, its union with the body being a temporary condition" (para. 10).

Descartes, however, famously advocated the dualist view of the mind (soul?) and body as distinct substances of the human being, while Spinoza claimed that the soul and body are one. Kant, nonetheless, concluded that the soul cannot be rationally explained (para. 7). Despite these philosophical and theological perspectives that suggest that in order for HNRs to have such a human-like existence 'of their own,' it is necessary that they are able to be self-aware. Reflection is the abstract creative reconsideration of recalled material, influenced by, but not bound by prior experience of some forms of experiences. 
In nursing, however, Rogers' (1970) Science of Unitary Human Beings has exceptionally underpinned quantum thought in concepts concerning human beings as energies and of the human body as their embodiment, suggesting that energies (as souls?) are sheathed/encased in the organic human body. As unitary human beings (Rogers, 1970) persons cannot be reduced to parts nor divided, are pandimensional energy fields, whose patterns manifest specific characteristics of being whole, and thereby are unpredictable from the knowledge of the parts. Persons are unitary wholes with unique features that cannot be supposed by viewing, labeling, or summarizing the parts (Fawcett \& De Santo-Madeya, 2013)). This viewpoint interprets the soul and energies as alike, incorporating the philosophical and theoretical expressions of 'person-wholeness' based on the human science view of human beings as greater than and different from the summated parts.

In Boykin and Schoenhofer's (2001) Nursing as Caring theory of nursing, a central assumption is that all persons are caring by virtue of their humanness. This assumption nurtures the essentiality of expressions of humanness that not one single facet (of being human) is standardized to describe "persons as caring". Inasmuch as it is the manifestation of caring that can be appropriated as caring, substantiated by philosophical, theoretical and theological views, these conspiring perspectives apparently can make real the possibility or probability of HNRs that are capable of manifesting caring. This possibility can be established by arguing that when manifestations of caring are recognized, the entity (that manifests caring) can probably be a caring person. From this affirmation, persons as human beings, as biological beings, as energies, souls, and as entity altogether can be declared the basis for the revolutionary structure of HNRs attesting to the likely provision of their capabilities to participate in caring practices rather than simply as programmed to perform.

\section{Is a HNR a Tool With Caring Function or a Nurses' Colleague?}

As an expression of love, concern and/or regard that is in the realm of human expression and that is "real" or experienced as caring, why is it necessary to assert that the humanoid is manifesting caring? As Schoenhofer queried, "as long as the assertion is with some philosophical and theoretical support that essentially declares that a humanoid can manifest caring, isn't that far enough?" (Personal communication email, 2018). Expressions of caring by persons who are appreciated as caring are substantiated by philosophical, theoretical and theological views, thereby making these manifestations 'of' caring.

Caring in nursing is the relationship between nurses and those whom they nurse grounded in specific caring science perspectives. Describing the essence of caring within nursing illuminates the person manifesting ingredients of caring (Mayeroff, 1971) and having attributes of caring (Roach, 2002). As a concept, caring can be appreciated as a verb, an adjective, or a noun, often creating a mystifying understanding of being 'caring in nursing.' While ingredients and attributes of caring guide the description of what is a caring person, it is caring as noun that is studied within the science of caring. As such, expressions of caring between the nurse and person being nursed (Boykin \& Schoenhofer, 2001) enhance living the meanings of their own lives. Therefore, with conditions of caring between the nurse and nursed, and the aspects and attributes of caring, will HNRs be able to manifest themselves as caring? As challenging as this statement may seem to be, the possibility and likelihood continue to remain plausible. Therefore, how will caring in nursing be appreciated, known, and practiced with HNRs as participant, or will HNRs be appreciated as nursing 'tools' with caring functions?

\section{Do Nurses 'Think Nursing' and Does It Matter?}

Concerns about humanness, caring in nursing, and the technologies of health care while promoting the appreciation of wholeness of persons as persons are highlighted as integral to HNR development and 'thinking nursing'. Currently, a common and traditional appreciation of caring practice in nursing consists of caring demonstrated through skillfulness in the completion of procedures specifically as nursing interventions. This imagery seems to illuminate nursing practice as perpetuated in a positivist philosophical viewpoint. It is the researchers' stance that completion of procedures is not in itself a sufficient end goal for nursing practice; yet frequently, within the traditional nursing process of assessment, planning, intervention and evaluation, nursing practice is popularly guided by a prescriptive or predictive practice. As Locsin and Purnell (2009) expressed, the ideal of caring in the practice of nursing ought to involve theoretically-based engagements informed by intelligent know-how.

The future of caring in nursing, with the advent of technological marvels such as HNRs, is in need of re-envisioning, founded on advances in technologies, given the impact that the influence of robotics and computer science has on how caring in nursing can be expressed and practiced. As within the Anthropocene era, (Monastersky, 2015) human beings dominate the global technological world, and therefore human beings become important protagonists in the expressions of humanness as their responsibilities for the world involve technological advances. 
Since it may be assumed that nursing engagement involving human nurses implicates higher thinking functions, HNRs will have to be highly technological so that they can effectively participate in encounters that are vital to the functioning feature of nursing practice, even those requiring higher thinking functions. HNRs must be able to communicate with human persons particularly regarding human health care (Huston, 2013). In this environment, Tanioka et al (2017) declared that valuing proficiency as a characteristic function of nurses should demand and enhance healthcare efficiency and quality.

In "thinking nursing," there are three theories of nursing proposed which support the ideal of caring in nursing as influential to HNR development towards manifesting caring. In Boykin and Schoenhofer's (2001) theory of Nursing as Caring, nursing situations are those shared experiences in which the caring between nurse and nursed enhances meaningful living as caring persons. This shared lived experience grounds Locsin's (2005) view of technological engagement, in which nursing occurs as co-created moments within nursing encounters within the Universal Technological Domain (UTD®) (Locsin \& Purnell, 202015), a domain in which all nursing in a technological world can take place. The nursing encounter is the simultaneous experiencing between the nurse and person being nursed in the process of knowing persons as caring within technological knowing, mutual designing, and participative engaging.

Furthermore, in Tanioka's (2017), Transactive Relationship Theory of Nursing, (TRETON) the technological and mutual engagement between the humanoid nurse robot and human nurses, or with HNRs and other HNRs simultaneously acknowledges a futuristic perspective. With views and theoretical framing derived from both Boykin and Schoenhofer's (2001). Nursing as Caring theory, and Locsin's (2005), (Locsin, 2016a), (Locsin, 2016b), (Locsin, 2017), theory of Technological Competency as Caring in Nursing, Tanioka's visioning of nursing engagement between human-to-machine, and human-to-human are prominent influences in the rethinking and re-envisioning of nursing as integral to the future practice of caring in nursing with intelligent machines. Concerns about humanness, caring in nursing, and the technologies of health care while promoting the appreciation of wholeness of persons as persons are highlighted as integral to 'thinking nursing'.

\section{Future Nursing Practice}

Many people make good use of technologies. However, the general public, and the elderly may not be adequately knowledgeable about the uses of sophisticated technologies in health care today. A critical and necessary consideration is to take time to introduce contemporary (and future) technologies, such as HNRs, especially in the patient care arena. Nonetheless, with time spanning intergenerational gaps, valuing technologies can be diverse. For example, Peck in 1992 declared that the main saving factor in nursing is the 'tender loving care' or TLC. If TLC is an expression of nursing and the co-created caring between the nurse and nursed in the nursing encounter, and if the science of caring grounds nursing practice, the futurist's question remains: Is the engagement of human-to-human or humanoid machines-to-humans with caring a core attribute of our nursing practice? (Locsin \& Ito, 2018)

Furthermore, Locsin and Ito referenced Mesquita, Zamarioli, and Carvalho's study of robots in healthcare in which thirty-five patents were found, of which $40 \%$ were focused on creating robots for immobile patients; $28.5 \%$ on robots to help perform daily activities; $23 \%$ for assessing physiological functioning of patients; and $8.5 \%$ for participation in rehabilitation. Locsin and Ito (2018) further declared that the possibilities of programming nursing tasks cleared the way for the invention of mechanical technologies including the mechanization of 'nurses' like automatons, a prospect that is undesirable.

Current developments of HNRs with varying types of technologies that influence human health care may not remain a distant reality (Tanioka, et al, 2017). Since 2015, with self-contained robots with a much improved physicality and likeness to human beings, the continuing recognition of nursing as task-completion is increasing the possibilities of HNRs with functions relevant to caring (Locsin \& Ito, 2018). Even though development of HNRs as idealized and programmed machines with AI is currently limited, the probability of their development clearly exists. Thus, it will likely be necessary for human beings to coexist with HNRs so that human nurses and HNRs are able to practice nursing in mutual engagements with shared roles, perhaps in institutional settings such as hospitals where deliberate caring practice is shared with patients based on human-to-human trusting relationships.

The implications of HNR functions are manifold, moving Locsin and Ito (2018) to describe the following scenario in introducing humanoid nurse robot care environments:

A patient walks into the Emergency Department (ED) and sits in front of the "triage nurse"-a computer that uses advanced algorithms to ask questions based on the patient's answers. A robot draws the patient's blood. Another one computes real-time nurse schedules and bed availability to decide if the patient can be admitted. "Follow 
me" the robot says (Glauser, 2017, p. 24).

In future HNR technologies, Locsin and Ito (2018) also affirm that artificial super intelligence provides the opportunity for HNRs to engage in caring well into the future of nursing practice. Technological machines that simply perform tasks that nurses do situate significant debates driving the functionality issues of intelligent machines.

Perhaps as future computers will be faster and have more advanced capabilities than human beings, HNRs will become the ultimate healthcare robots performing more complex functions than those which can now be achieved (Locsin \& Ito, 2018). Therefore, how will human nurses and HNRs practice nursing together? As caring is manifested by HNRs, what will it be like for human nurses to practice nursing alongside HNRs, or with persons who are technologically enhanced such as cyborgs or cybernetic organisms, particularly in this unique 'nurse-nursed' relationship? In order for HNRs to perform like human beings with emotion and sensitivity, it is important to consider the following viewpoints.

In today's highly developed social engagements, technologies such as computers, data bases, the Internet, and cloud server provide elemental features for human beings. These technological devices are now common requisites for human beings living in a human social group. HNRs can possess human sensibilities, sharing memories with significant persons (friends, patients, families) while manifesting their caring.

In these situations, HNR functions may require miniature alternative databases, serving as the "new mechanism" of storing information in which a secure database is needed so that the HNR can intelligently manifest technological competency. Furthermore, in developing the HNR concerns exist at to what security processes can be encoded in case the Internet system fails or stops. Will it continue to demonstrate sufficient communicative ability? What about the "leakage" of personal information (accumulated in the cloud server) becoming a security problem? Actually, these may not be unique problems of HNRs, as human beings are also predisposed to violating secrecy rules, often considered a flaw and a feature of being human. Nevertheless, HNRs need to have competent functions which can secure information from unwanted invasion of privacy.

\section{Technological Competency as Caring in Nursing Theory}

The theory of Technological Competency as Caring in Nursing is founded on assumptions that give structure and meaning to the theory. Theoretical assumptions from the theory (Locsin, 2005), Locsin, 2016a), Locsin, 2017), Locsin \& Ito, 2018) define the elemental configuration guiding the requisite design and development of HNRs in being and becoming caring entities.

\section{- Persons are caring by virtue of their humanness (Boykin \& Schoenhofer, 2001)}

As caring persons, expressions of humanness are fundamental to human-human relationships. In the case of human-machine relationships, this expectation can be valid as well, manifested as expressions of caring. As humanness is an expression of caring persons, the distinct relationships of caring persons are made real. It is the element of the caring domain that influences the ontology of nursing, thereby allowing caring to be known in HNRs by virtue of human expressions and manifestations in the practice of nursing Locsin \& Ito, 2018).

- The ideal of wholeness is a perspective of oneness (Locsin \& Ito, 2018)

Persons are appreciated as whole beings without reference to composition of parts. HNRs capable of caring exhibit wholeness as manifestations of being, for example, the oneness of nurse-nursed engagement within the caring between, and the oneness in the togetherness of relating.

- Knowing persons is a multidimensional process (Locsin, 2005)

Illuminating the process through which a nurse and machine may 'transactively' relate with one another involves an affirming process in which caring 'persons' are engaged in mutual designing while recognizing the other as active participant in their care (Locsin \& Ito, 2018)

- Technologies of health and nursing are elements of caring (Locsin, 2005). Through technologies of health and nursing, persons are known more fully as wholes and active accomplices in their care, instead of as only objects of care (Locsin \& Ito, 2018)

- Nursing as a discipline and a professional practice (Boykin \& Schoenhofer, 2001). As the fundamental commitment in the scholarly practice of nursing founded on caring in nursing, advancing nursing as a discipline is realizing that it has its own unique body of knowledge as basis for its practice that is integral to human health and human care.

Directed towards the practice of nursing and nurses' sensibilities of embracing nursing practice, the theory of 
Technological Competency as Caring in Nursing (Locsin \& Purnell, 2015) proposed a unique process of nursing, engaging three distinctive processes as events in knowing persons as caring. These are technological knowing, mutual designing, and participative engaging. These processes occur all-at-once and are interacting with each other while fostering recognitions of nursing as practicing in synchrony with human to human and human to machine relatedness. Guided by these theoretical assumptions, the probability of HNRs being and becoming capable of manifesting caring is likely enhanced.

\section{How Can HNRs Manifest Being Caring?}

In addition to having accoutrements such as human-like physiognomies, programmed functionalities, voice and language, and sensory capabilities (Kanda, et al, 2004), HNRs must be able to feel and touch others as much as health care providers do (Tanioka, et al 2017) delicately, (Schmidt, Mael, Wurtz (2006) and without the fear of causing injury to others (Harper \& Virk, 2010). HNRs have to match the gaze of patients (Schmidt, Mael, Wurtz (2006), while reacting to patient's actions (Bicho, et al, 2010) HNRs have to talk with other people with ease, and must be able to understand and express patients' bio-behavioral responses and expressions of stress (Itoh, et al, 2006). With all these considerations, however, it has been established that $65 \%$ of human interactions are actually transmitted through non-verbal communication, such as through or by way of gestures, including facial expressions and body behaviors and timely responses during conversations (Birdwhistell, 1970; Vargas, 1986).

In the future, will natural language be dominant in the capabilities of HNRs in relating with human nurses and other HNRs? If $65 \%$ of human interactions is dominated by movement or gestures, will it still be language that remains the primary way of relating in conversations between humans and HNRs?

It is important to note that human interactions are often linked with aspects of being human. Mehrabian (1981) in a book entitled Silent Messages, declared that study participants assigned 55 percent of their observations to the speaker's body language, and another 38 percent to the tone and sound (musicality) of their voices. They only assigned seven per cent of their credibility assessment to the salesperson's actual words. These findings suggest that in patients and their families who express a dislike for a nurse, there may have been a very outspoken attitude that they did not like. As Curtis and Miller (1986) declared, human beings tend to like persons who seem to like them. Therefore, it seems apparent that nonverbal expression is a performance requirement for HNRs as well.

\section{The Practice Processes Central to Caring With Humanoid Nurse Robots}

The nursing processes proposed by Locsin and Purnell (2009) are co-created nursing encounters between human to human nurses or with HNRs who are participants in the care. The non-probabilistic feature (unpredictability) of human beings challenges the appreciation of technological competency as that of manifesting caring in nursing. HNRs will have the programmed ability to manifest behavior tantamount to caring. In technological knowing, technologies are used to know persons as persons, whole and complete in the moment, not needing to be fixed or made whole again. Mutual designing is the process in which the nurse and the one nursed (patient) together foster, affirm, support and celebrate persons as caring. Participative engaging is the simultaneous practice of relating with an 'other.' These are significant features of caring as evidenced by the continuing and repeating process of knowing persons as caring in which the nurse and nursed enhance living the meaning of one's own life.

In order to advance the performance of current and future HNRs, it is imperative that they have "desirable relationships with persons, to notice slight changes in their expressions, and in their tone of voice" (Fuji, et al, 2014, p. 349). Furthermore, HNRs can be capable of innovation and creative thought, action and transaction by virtue of highly advanced computers. These are also vital features of manifesting caring. HNRs can have the linguistic ability to manifest themselves with others through transactive relations.

Human persons maintain that they have the right to determine effective treatments and health care (Stahl, Coekelbergh, (2016). In the practice of nursing there are shared encounters between caring persons in co-created nursing moments within the universal technological domain (Locsin \& Purnell, 2015). These engagements foster the realization of future technological developments in robotics, artificial superintelligence, and viewpoints of being able to manifest caring. It is the value of human caring in nursing that becomes the impetus to the redefinition of nursing as a discipline of knowledge and practice profession within the technological world of the Anthropogenic era.

Van Wynsberghe (2013) expressed the importance of ensuring that [robot] design does not inhibit advancing the values and dignity of persons who are in a vulnerable and sensitive time in their lives. Therefore, nursing must be redefined in its ontology and epistemology, to avoid the prospect that the continuity of nursing practice 
becomes largely independent of what is the practice of nursing being manifested as caring.

\section{Conclusion}

Human nurses have qualities of humanness that may not always be expressed in nursing care situations. A person's humanness certainly includes an emotional content regardless of well-intentioned activity. For HNRs, however, to be more human-like and manifest caring in health care situations, they will need to show facial expressions and gestures as functionally imbued by heir humanness as much as possible.

Ultimately, notwithstanding HNRs being and becoming able to manifest caring, these questions remain "how will nursing practice advance, and human nurses be made central to human health and well-being with HNRs manifesting caring?"; and "is it important to know how human nurses will be able to accept revolutionary changes if and when HNRs will operate in healthcare settings?".

The thought that HNRs might be able to creatively manifest caring may be construed as esoteric, far-fetched, and perhaps improbable. The authors have presented philosophical and theoretical arguments supporting probabilities and possibilities for HNRs being and becoming able to manifest caring (or perhaps simply being appreciated as caring). Descriptions of nursing, caring, and the practice of nursing as 'knowing persons' are considered instrumental to this probability of HNRs being and becoming able to manifest caring. When nursing is the expression of the caring between the nurse and nursed enhancing personhood, it is quite essential that HNRs can and will be able to manifest caring, participating in human caring relations that in all matters foster an appreciated nursing caring practice.

\section{References}

Barrat, J. (2013). Our Final Invention. St. Martin's Press, New York.

Bicho, E., Louro, L., \& Erlhagen, W. (2010). Integrating verbal and nonverbal communication in a dynamic neural field architecture for human-robot interaction. Frontiers in Neurorobotics, $4,5$. https://doi.org/10.3389/fnbot.2010.00005

Birdwhistell, R.L. (1970). Kinesics and Context: Essays on Body Motion Communication. University of Pennsylvania Press, Philadelphia.

Boykin, A., \& Schoenhofer, S. (2001). Nursing as Caring: A Model for Transforming Practice. Jones and Bartlett, Sudbury, CT.

Curtis, R.C. (1986). Miller K. Believing another likes or dislikes you: Behaviors making the beliefs come true. Journal of Personality and Social Psychology, 51(2), 284-290. https://doi.org/10.1037/0022-3514.51.2.284

Fawcett, J., \& De-Santo-Madeya, S. (2013). Contemporary Nursing Knowledge: Analysis and Interpretations of Nursing Models and Theories. F.A, Davis, Philadelphia, PA, USA.

Fuji, S., Yasuhara, Y., Huang, S., Tanioka, T., \& Locsin, R. (2014). Discussion of nursing robot's capability and ethical issues. Information, 17(1), 349-353.

Glauser, W. (2017). Artificial intelligence, automation, and the future of nursing. Canadian Nursing, 113(3), 24-26.

Harper, C., \& Virk, G. (2010). Towards the development of international safety standards for human robot interaction. Int J of Soc Robotic, 2, 229. https://doi.org/10.1007/s12369-010-0051-1

Huston, C. (2013). The impact of emerging technology on nursing care: warp speed ahead. The Online Journal of Issues in Nursing, 18(2), Manuscript 1. https://doi.org/10.3912/OJIN.Vol18No02Man01

Itoh, K., Miwa, H., Nukariya, Y., Zecca, M., Takanobu, H., Roccella, S., ... Takanishi, A. (2006). Development of a bioinstrumentation system in the interaction between a human and a robot. IEEE/RSJ International Conference on Intelligent Robots and Systems. https://doi.org/10.1109/IROS.2006.281941

Kanda, T., Ishiguro, H., Imai, M., \& Ono, T. (2004, November). Development and evaluation of interactive humanoid robots. Proceedings of the IEEE, 92(11). https://doi.org/10.1109/JPROC.2004.835359

Kurzweil, R. (2005). The Singularity Is Near: When Humans Transcend Biology. Viking Books, New York.

Locsin, R, \& Purnell, M. (2009). A Contemporary Nursing Process: The (Un) bearable Weight of Knowing in Nursing. Springer Publishing, New York, NY.

Locsin, R. (2005). Technological Competency as Caring in Nursing. Sigma Theta Tau International Press, Indianapolis, IN. 
Locsin, R. (2016a). Technological Competency as Caring in Nursing. Silliman University Press, Dumaguete City, Philippines.

Locsin, R. (2016b). The theory of Technological Competency as Caring in Nursing: guiding nursing and health care. Shikoku Acta Medica, 72(5, 6), 163-70.

Locsin, R. (2017). The co-existence of technology and caring in nursing in the theory of Technological Competency as Caring in Nursing. Journal of Medical Investigation, 64(1, 2), 160-164.

Locsin, R., \& Purnell, M. (2015). Advancing the theory of Technological Competency as Caring in Nursing: the universal technological domain. International Journal for Human Caring, 19(2), 50-54. https://doi.org/10.20467/1091-5710-19.2.50

Locsin, R.C., \& Ito, H. (2018). Can humanoid nurse robots replace human nurses? Journal of Nursing, 5(1). Retrieved from http://www.hoajonline.com/nursing/2056-9157/5/1

Mayeroff, M. (1971). On Caring. Harper-Perennial, New York.

Mehrabian, A. (1981). Silent Messages: Implicit Communication of Emotions and Attitudes (2nd ed). Wadsworth Publishing, Belmont, CA.

Mesquita, A.C., Zamarioli, A.M., \& Carvalho, E.C. (2016). The use of robots in nursing care practices: an exploratory-descriptive study. Online Brazilian Journal Nursing, 15(3), 404-413. https://doi.org/10.17665/1676-4285.20165395

Monastersky, R. (2015). Anthropocene: the human age. Nature, 519(7542), 144-147. https://doi.org/10.1038/519144a

Peck, M.L. (1992). The future of nursing in a technological world. Holistic Nursing Practice, 10(2), 183-191. https://doi.org/10.1177/089801019201000208

Ren, F., \& Matsumoto, K. (2017). Natural language processing capabilities required for humanoid nursing robots, Chapter 8. In Tanioka T. et al. (eds.), Nursing Robots: Robotic Technology and Human Caring for the Elderly. Fukuro Publishing, Okayama, Japan.

Roach, S. (2002). Caring: The Human Mode of Being: A Blueprint for the Health Professions (2nd Ed). Canadian Healthcare Association Press, Ottawa. (Copy from Archives of Caring in Nursing, Christine E. Lynn College of Nursing, Florida Atlantic University, ARC-005. Sister M. Simone Roach Archives, 1958-2005. Used with permission.)

Robotic devices for nursing care. (2016). Retrieved January 29, 2018, from http://robotcare.jp/wp-content/uploads/2016/11/ROBOT-CARE-pamphlet_eng.pdf

Rogers, M. (1970). Theoretical Basis of Nursing. F.A. Davis, New York.

Savage, N. (2017). Quantum computers compete for "supremacy". Scientific American. Retrieved from https://www.scientificamerican.com/article/quantum-computers-compete-for-supremacy/

Schmidt, P.A., Maël, A., \& Würtz, R.P. (2006). A sensor for dynamic tactile information with applications in human-robot interaction and object exploration, Robotics and Autonomous Systems, 54(12), 1005-1014. https://doi.org/10.1016/j.robot.2006.05.013

Schoenhofer. (2018). Personal Communication.

"Soul." Encyclopedia Britannica. (2017). Encyclopedia Britannica. Retrieved from https://www.britannica.com/topic/ soul-religion-and-philosophy

Stahl, B., \& Coeckelbergh, M. (2016). Ethics of healthcare robotics: towards responsible research and innovation. Robotics and Autonomous Systems, 86, 152-161. https://doi.org/10.1016/j.robot.2016.08.018

Tanioka, T. (2017). The development of the transactive relationship theory of nursing. Nursing Robots: Robotic Technology and Human Caring for the Elderly. Fukuro Publishing, Okayama, Japan.

Tanioka, T., Osaka, K., Locsin, R., Yasuhara, Y., \& Ito, H. (2017). Recommended design and direction of development for humanoid nursing robots perspective from nursing researchers. Intelligent Control and Automation, 8, 96-110. https://doi.org/10.4236/ica.2017.82008

van Wynsberghe, A. (2013). Designing robots for care: care centered value-sensitive design. Science and Engineering Ethics, 19(2), 407-433. https://doi.org/10.1007/s11948-011-9343-6

Vargas, M.F. (1986). Louder Than Words: An Introduction to Nonverbal Communication. Iowa State Press, Iowa. 
Wagoner, A., \& Matson, E. (2015). A robust human-robot communication system using natural language for HARMS. Procedia Computer Science, 56, 119-126. https://doi.org/10.1016/j.procs.2015.07.178

Wilson, M.D. (1976). Descartes: the epistemological argument for mind-body distinctness. Nous, 10(1), 3-15. https://doi.org/10.2307/2214469

Yonck, R. (2017). The Heart of the Machine: Our Future in a World of Artificial Emotional Intelligence. Arcade Publishing, New York.

\section{Copyrights}

Copyright for this article is retained by the author(s), with first publication rights granted to the journal.

This is an open-access article distributed under the terms and conditions of the Creative Commons Attribution license (http://creativecommons.org/licenses/by/4.0/). 